\title{
Wilcoxon signed rank test for imprecise observations
}

\author{
V.S.Vaidyanathan ${ }^{1}$ \\ ${ }^{I}$ Department of Statistics, Pondicherry University, Puducherry - 605 014, India
}

\begin{abstract}
This article extends the Wilcoxon signed rank test for testing whether the median of a population is a specified constant by treating the observations as imprecise values. The test procedure is developed by using the concepts of "Credibility Theory" for studying the behavior of fuzzy phenomena. Numerical illustration of the proposed test is provided by representing the observations as trapezoidal fuzzy numbers.
\end{abstract}

Keywords: Credibility measure, fuzzy number, fuzzy ordering, Wilcoxon test.

\section{Introduction}

Statistical tests are used to draw inference about the underlying population distribution based on certain hypothesis concerning one or more parameters of the distribution. The basic assumption in these tests is that the underlying probability distribution of the population is known except perhaps for the parameters involved. Such tests are generally referred to as parametric tests. Neyman-Pearson based approach and likelihood based approach are two popular approaches used in carrying out parametric tests. Details about these approaches are available in Lehmann and Romano [1]. Sometimes no information is available even about the functional form of the probability distribution except the information that it is continuous. In such situations, non-parametric approach is usually used. Gibbons and Chakraborti [2] contain details about various non-parametric tests. One popular non-parametric test that is used to test whether the median of a population is a specified constant is Wilcoxon signed rank test. This test makes use of the ranks of the signed differences of the sample observations from the median.

Generally, in dealing with the above tests, it is assumed that the observations are accurately measured or expressed. However, in practice there are situations wherein observed values are imprecise or expressed in vague terms. This happens especially with data that are measured on a continuous scale because exact measurement is not possible. Examples are measurement on body temperature, physical measurements like length, height, weight etc. Fuzzy set theory proposed by Zadeh [3] can be used in these cases to express the measured values as fuzzy numbers with pre-defined membership functions. Zadeh's theory has been applied in developing statistical techniques for univariate and multivariate data. Kruse [4] discusses some methods for statistical estimation. Kruse and Meyer [5], Manton et al. [6] and Viertl [7] have developed statistical methods for imprecise data sets. Testing of statistical hypothesis with fuzzy information can be found in Casals et al. [8], Romer and Kandel [9] and Grzegorzewski [10]. Chen [11] has proposed statistical inference procedures for imprecise data using possibility theory. Recently Liu [12] has developed a new theory to deal with fuzzy phenomena called credibility theory. This theory is based on certain axioms and has nice mathematical properties. Details about credibility theory and its associated results can be found in Liu [12]. The primary advantage of credibility theory approach is that it does not make use of $\alpha$-cuts for performing arithmetic and relational operations. Based on credibility theory, Vaidyanathan [13] has proposed a correlation measure between triangular fuzzy variables. Recently Hesamian and Taheri [14] have developed non-parametric sign test for imprecise data using credibility theory. Also Taheri and Hesamin [15] have proposed a generalization of the Wilcoxon signed rank test using the concept of fuzzy random variables in which the test statistic as well as the critical value is fuzzy.

In this article, an extension of Wilcoxon signed rank test for imprecise data is proposed using the concepts of credibility theory. The rest of the paper is organized as follows. In Section 2, certain important concepts, definitions and results based on credibility theory are discussed. Section 3 contains a brief description about Wilcoxon signed rank test. Section 4 describes the proposed Wilcoxon test for imprecise data. In Section 5, a numerical illustration of the proposed test is given. Section 6 concludes the article with discussion and future work.

\section{Preliminaries}

To model imprecise values using fuzzy sets, Liu [12] has developed a mathematical theory based on axioms called 'Credibility Theory'. This section contains the definitions of certain important concepts and some results based on credibility theory. For detailed proofs of the results, readers may refer Liu [12]. 


\section{Credibility measure}

Let $\Theta$ be a nonempty set, and $\mathrm{P}$ the power set of $\Theta$ i.e., the largest $\sigma$-algebra over $\Theta$. Each element in $\mathrm{P}$ is called an event. The set function $\mathrm{Cr}$ is called a credibility measure if it satisfies the following axioms.

Axiom 1: (Normality) $\operatorname{Cr}\{\Theta\}=1$.

Axiom 2: (Monotonicity) $C r\{A\} \leq C r\{B\}$ whenever $A \subset B$.

Axiom 3: (Self duality) $\operatorname{Cr}\{A\}+\operatorname{Cr}\left\{A^{C}\right\} \equiv 1$ for any event $A$.

Axiom 4: (Maximality) $\operatorname{Cr}\left\{\bigcup_{i} A_{i}\right\}=\sup _{i} \operatorname{Cr}\left\{A_{i}\right\}$ for any event $\left\{A_{i}\right\}$ with

$$
\sup _{i} \operatorname{Cr}\left\{A_{i}\right\}<0.5 \text {. }
$$

$\operatorname{Cr}\{A\}$ indicate the credibility that the event $A$ will occur. The triplet $(\Theta, \mathrm{P}, C r)$ is called a credibility space.

Fuzzy variable: A fuzzy variable is a (measurable) function from the credibility space $(\Theta, P, C r)$ to the set of real numbers.

Remark: Any function of fuzzy variables defined on the same credibility space is again a fuzzy variable i.e., the sum or product of two or more fuzzy variables is again a fuzzy variable.

Membership function: The membership function $\mu$ of a fuzzy variable $\xi$ defined on the credibility space $(\Theta, \mathrm{P}, C r)$ is given by $\mu(x)=\min (2 C r\{\xi=x\}, 1), x \in \mathfrak{R}$.

Remark: Membership function represents the degree that the fuzzy variable $\xi$ takes on some prescribed value and it always takes values in the interval [0,1]. Depending on the shape of the curve of membership functions, fuzzy variables are defined appropriately. Often used fuzzy variables are triangular and trapezoidal fuzzy variable.

Triangular fuzzy variable: A fuzzy variable fully determined by the triplet $(a, b, c)$ of crisp numbers with $(a<b<c)$ and whose membership function is given by

$$
\mu(x)=\left\{\begin{aligned}
\frac{x-a}{b-a}, & \text { if } a \leq x \leq b \\
\frac{x-c}{b-c}, & \text { if } \quad b \leq x \leq c \\
0, & \text { otherwise }
\end{aligned}\right.
$$

is called a triangular fuzzy variable. If $b-a=c-b$ then the triangular fuzzy variable is said to be symmetric.

Trapezoidal fuzzy variable: A fuzzy variable fully determined by the quadruplet $(a, b, c, d)$ of crisp numbers with $(a<b<c<d)$ and whose membership function is given by

$$
\mu(x)=\left\{\begin{aligned}
\frac{x-a}{b-a}, & \text { if } \quad a \leq x \leq b \\
1, & \text { if } \quad b \leq x \leq c \\
\frac{x-d}{c-d}, & \text { if } \quad \mathrm{c} \leq x \leq d \\
0, & \text { otherwise }
\end{aligned}\right.
$$

is called a trapezoidal fuzzy variable. If $b-a=d-c$ then the trapezoidal fuzzy variable is said to be symmetric. Also if $b=c$ then the trapezoidal fuzzy variable reduces to a triangular fuzzy variable. 
Credibility Inversion theorem: Let be $\xi$ be fuzzy variable with membership function $\mu$. Then for any set $B$ of real numbers, $\operatorname{Cr}\{\xi \in \mathrm{B}\}=\frac{1}{2}\left(\sup _{\mathrm{x} \in \mathrm{B}} \mu(\mathrm{x})+1-\sup _{\mathrm{x} \in \mathrm{B}} \mathrm{c} \mu(\mathrm{x})\right)$.

Credibility distribution: The credibility distribution $\Phi: \mathfrak{R} \rightarrow[0,1]$ of a fuzzy variable $\xi$ is defined by $\Phi(x)=\operatorname{Cr}\{\theta \in \Theta \mid \xi(\theta) \leq x\}$.That is, $\Phi(x)$ is the credibility that the fuzzy variable $\xi$ takes a value less than or equal to $x$.

Expected value of a fuzzy variable: The expected value of a fuzzy variable $\xi$ denoted by $E(\xi)$ is defined as $E(\xi)=\int_{0}^{+\infty} C r\{\xi \geq r\} d r-\int_{-\infty}^{0} C r\{\xi \leq r\} d r$ provided that atleast one of the integrals is finite.

Variance of a fuzzy variable: The variance of a fuzzy variable $\xi$ with finite expected value $e$ and denoted by $V(\xi)$ is defined as $V(\xi)=E(\xi-e)^{2}=\int_{0}^{+\infty} C r\left\{(\xi-e)^{2} \geq r\right\} d r$. Variance of a fuzzy variable provides a measure of spread of the membership function around its expected value.

Ordering fuzzy variables: Let $\xi$ and $\eta$ be two fuzzy variables defined on the credibility space $(\Theta, P, C r)$. The ordering of $\xi$ and $\eta$ is defined as follows (see Li et al. [16]):

1. $\xi \succ \eta$ if and only if $E[\xi]>E[\eta]$ or $E[\xi]=E[\eta]$ and $V(\xi)<V(\eta)$.

2. $\xi \prec \eta$ if and only if $E[\xi]<E[\eta]$ or $E[\xi]=E[\eta]$ and $V(\xi)>V(\eta)$.

3. $\xi \sim \eta$ if and only if $E[\xi]=E[\eta]$ and $V(\xi)=V(\eta)$.

Thus $\xi$ is greater than or equal to $\eta$ if and only if $\xi \succ \eta$ or $\xi \sim \eta$ and $\xi$ is lesser than or equal to $\eta$ if and only if $\xi \prec \eta$ or $\xi \sim \eta$.

\section{Wilcoxon Singed rank test}

This test is used to determine whether the median $\mu$ of a population (taking continuous values) is a specified constant say $\mu_{0}$ i.e., $\mathrm{H}: \mu=\mu_{0}$ based on random observations from the population. The test is carried out by determining the ranks of the absolute differences of the observations from $\mu_{0}$, namely $\mathrm{D}$, with the assumption that no D is zero, and no two D's have the same magnitude. The ranks are assigned + and - signs depending on whether the difference is positive or negative. Let $\mathrm{T}^{+}\left(\mathrm{T}^{-}\right)$denote the absolute sum of positive (negative) signed ranks. If there are $\mathrm{n}$ observations, then sum of $\mathrm{T}^{+}$and $\mathrm{T}^{-}$will be equal to $\frac{\mathrm{n}(\mathrm{n}+1)}{2}$. Therefore, under $\mathrm{H}, \mathrm{T}^{+}\left(\mathrm{T}^{-}\right)$is expected to be around $\frac{\mathrm{n}(\mathrm{n}+1)}{4}$. Thus if $\mathrm{T}^{+}$is much different from $\frac{\mathrm{n}(\mathrm{n}+1)}{4}$, then the hypothesis $\mathrm{H}$ is likely to be not true. $\mathrm{T}^{+}$is known as Wilcoxon statistic and its probability distribution under $\mathrm{H}$ is given by $\mathrm{P}\left[\mathrm{T}^{+}=\mathrm{t} \mid \mathrm{H}\right]=\frac{\mathrm{r}_{\mathrm{t}}}{2^{\mathrm{n}}}$, where $\mathrm{r}_{\mathrm{t}}$ is the number of ways of assigning + and - signs to the ranks $1,2, \ldots, \mathrm{n}$ such that $\mathrm{T}^{+}=\mathrm{t}$. Thus a decision to accept $\mathrm{H}$ is made by computing the above probability. However for large $\mathrm{n}$, one may use the standardized $\mathrm{T}^{+}$statistic given by $\mathrm{Z}=\frac{\mathrm{T}^{+}-\mathrm{E}\left(\mathrm{T}^{+}\right)}{\sqrt{\mathrm{V}\left(\mathrm{T}^{+}\right)}} \sim \mathrm{N}(0,1)$, with $\mathrm{E}\left(\mathrm{T}^{+}\right)=\frac{\mathrm{n}(\mathrm{n}+1)}{4}$ and $\mathrm{V}\left(\mathrm{T}^{+}\right)=$ $\frac{\mathrm{n}(\mathrm{n}+1)(2 \mathrm{n}+1)}{24}$. More details about the test can be found in Rajagopalan and Dhanavanthan [17].

\section{Proposed Wilcoxon test}

In the proposed test, the sample observations are assumed to be fuzzy numbers. The hypothesis to be tested is $\mathrm{H}: \tilde{\mu}=\widetilde{\mu_{0}}$ where $\tilde{\mu}$ denote the population median and $\widetilde{\mu_{0}}$ is its specified value (also a fuzzy number). Thus the hypothesis to be tested is also fuzzy. Let there be $\mathrm{n}$ sample observations denoted by $\xi_{1}, \xi_{2}, \ldots, \xi_{\mathrm{n}}$ with corresponding membership functions $\mu\left(\xi_{1}\right), \mu\left(\xi_{2}\right), \ldots, \mu\left(\xi_{n}\right)$. The procedure to carry out the test is given in the following steps.

Step 1: Obtain the difference $D_{i}$ of the observation $\xi_{i}$ from $\widetilde{\mu_{0}}$ i.e., $D_{i}=\xi_{i}-\widetilde{\mu_{0}}$,

$\mathrm{i}=1,2, \ldots, \mathrm{n}$ and determine the absolute value of the difference.

Step 2: Find the expectation and variance of the absolute value of the differences. 
Step 3: Arrange in increasing order the absolute value of the differences using the method of ordering fuzzy numbers given in section 2 after omitting those observations that have equal or zero absolute difference.

Step 4: Rank the absolute differences based on the ordered arrangement obtained in Step 3 and assign + and signs depending on whether the difference is positive or negative.

Step 5: Determine $\mathrm{T}^{+}$the sum of positive signed ranks.

Step 6: Calculate the p-value of the test using the probability distribution of $T^{+}$i.e., $P\left[T^{+}=t \mid H\right]=\frac{r_{t}}{2^{n}}$, where $r_{t}$ is the number of ways of assigning + and - signs to the ranks $1,2, \ldots, \mathrm{n}$ such that $\mathrm{T}^{+}=\mathrm{t}$.

Step 7: Reject the hypothesis $\mathrm{H}: \tilde{\mu}=\widetilde{\mu_{0}}$ at level $\delta$ if $\mathrm{P}\left[\mathrm{T}^{+}=\mathrm{t} \mid \mathrm{H}\right]<\delta$.

For large samples, the standardized $\mathrm{T}^{+}$statistic may be used. It is emphasized that in the proposed test the hypothesis is fuzzy but the test statistic is crisp. The advantage of the above test is that it does not depend on the $\alpha$-cut approach for performing arithmetic and relational operations on fuzzy numbers thereby making the calculations simpler.

\section{Numerical illustration}

As an illustration of the proposed test, the example of a fuzzy data set mentioned in Hesamian and Taheri [14] is considered. The data relate to the performance evaluation of workers in an aerospace firm made on a fuzzy scale by expressing the scores as trapezoidal fuzzy numbers. The scores pertaining to a random sample of 16 workers is given below.

Table 1: Performance scores of workers

\begin{tabular}{|c|c|c|c|}
\hline Sample No. & Score & Sample No. & Score \\
\hline 1 & $(60,65,65,70)$ & 9 & $(60,65,65,75)$ \\
\hline 2 & $(60,65,65,75)$ & 10 & $(75,80,85,90)$ \\
\hline 3 & $(80,85,85,90)$ & 11 & $(80,90,90,100)$ \\
\hline 4 & $(55,60,70,75)$ & 12 & $(40,50,55,75)$ \\
\hline 5 & $(65,70,75,80)$ & 13 & $(50,55,55,70)$ \\
\hline 6 & $(60,75,75,80)$ & 14 & $(60,65,65,70)$ \\
\hline 7 & $(70,80,80,90)$ & 15 & $(70,75,75,80)$ \\
\hline 8 & $(75,85,85,90)$ & 16 & $(60,65,75,80)$ \\
\hline
\end{tabular}

The hypothesis to be tested is $\mathrm{H}: \tilde{\mu}=(70,80,80,90)$ where $\tilde{\mu}$ denote the median score of the workers in the firm. To implement the proposed test, the following results about trapezoidal fuzzy numbers are used.

Result 1: Let $\xi=(\mathrm{a}, \mathrm{b}, \mathrm{c}, \mathrm{d})$ denote a trapezoidal fuzzy number. Then the negative of $\xi$ is given by $-\xi=(-d,-c,-b,-a)$. Also $\xi$ is said to be positive if a $>0$ and negative if $d<0$.

Result 2: Let $\xi_{1}=(\mathrm{a}, \mathrm{b}, \mathrm{c}, \mathrm{d})$ and $\xi_{2}=(\mathrm{m}, \mathrm{n}, \mathrm{p}, \mathrm{q})$ denote two trapezoidal fuzzy numbers. Then the difference between $\xi_{1}$ and $\xi_{2}$ is given by $\xi_{1}-\xi_{2}=(a-q, b-p, c-n, d-m)$.

Result 3: The expected value of a trapezoidal fuzzy number $\xi=(a, b, c, d)$ is given by $E(\xi)=\frac{a+b+c+d}{4}$ and its variance is given as below.

Case 1: When $\alpha=\beta, V(\xi)=\frac{3(\mathrm{~b}-\mathrm{a}+\beta)^{2}+\beta^{2}}{24}$

Case 2: When $\alpha>\beta, V(\xi)=\left\{\begin{array}{l}\frac{1}{6}\left[-\frac{(b-m)^{3}}{\beta}-\frac{(a-\alpha-m)^{3}}{\alpha}+\frac{(b \alpha+\alpha \beta-m(\alpha+\beta))^{3}}{\alpha \beta(\alpha-\beta)^{2}}\right], \quad a-m<0 \\ \frac{1}{6}\left[\frac{(a-m)^{3}}{\alpha}-\frac{(b-m)^{3}}{\beta}-\frac{(a-\alpha-m)^{3}}{\alpha}+\frac{(b \alpha+\alpha \beta-m(\alpha+\beta))^{3}}{\alpha \beta(\alpha-\beta)^{2}}\right], a-m \geq 0\end{array}\right.$

Case 3: When $\alpha<\beta, V(\xi)=\left\{\begin{array}{l}\frac{1}{6}\left[\frac{(a-m)^{3}}{\alpha}+\frac{(b+\beta-m)^{3}}{\beta}+\frac{(b \alpha+\alpha \beta-m(\alpha+\beta))^{3}}{\alpha \beta(\alpha-\beta)^{2}}\right], \quad b-m>0 \\ \frac{1}{6}\left[\frac{(a-m)^{3}}{\alpha}-\frac{(b-m)^{3}}{\beta}+\frac{(b+\beta-m)^{3}}{\beta}+\frac{(b \alpha+\alpha \beta-m(\alpha+\beta))^{3}}{\alpha \beta(\alpha-\beta)^{2}}\right], b-m \leq 0\end{array}\right.$

where $\alpha=b-a ; \beta=d-c$ and $m=E(\xi)$.

The following table shows the difference (D) of each sample observation from the median $\tilde{\mu}$ given under $\mathrm{H}$, the absolute difference $(|\mathrm{D}|)$ and the corresponding expected value $(\mathrm{E})$, variance $(\mathrm{V})$ and rank (ignoring tied values). 
Table 2: Ranks based on absolute difference

\begin{tabular}{|c|c|c|c|c|c|}
\hline Sample & $\mathrm{D}$ & $|\mathrm{D}|$ & $E$ & $\mathrm{~V}$ & Rank \\
\hline 1 & $(-30,-15,-15,0)$ & $(-30,-15,-15,0)$ & -15 & 121.875 & $* * *$ \\
\hline 2 & $(-30,-15,-15,5)$ & $(-30,-15,-15,5)$ & -13.75 & 3868.321398 & $* * *$ \\
\hline 3 & $(-10,5,5,20)$ & $(-10,5,5,20)$ & 5 & 121.875 & 10 \\
\hline 4 & $(-35,-20,-10,5)$ & $(-35,-20,-10,5)$ & -15 & 121.875 & $* * *$ \\
\hline 5 & $(-25,-10,-5,10)$ & $(-25,-10,-5,10)$ & -7.5 & 121.875 & 5 \\
\hline 6 & $(-30,-5,-5,10)$ & $(-30,-5,-5,10)$ & -7.5 & 1453.75 & 4 \\
\hline 7 & $(-20,0,0,20)$ & $(-20,0,0,20)$ & 0 & 216.6666667 & 7 \\
\hline 8 & $(-15,5,5,20)$ & $(-15,5,5,20)$ & 3.75 & 916.2109375 & 9 \\
\hline 9 & $(-30,-15,-15,5)$ & $(-30,-15,-15,5)$ & -13.75 & 3868.321398 & **** \\
\hline 10 & $(-15,0,5,20)$ & $(-15,0,5,20)$ & 2.5 & 121.875 & 8 \\
\hline 11 & $(-10,10,10,30)$ & $(-10,10,10,30)$ & 10 & 216.6666667 & 11 \\
\hline 12 & $(-50,-30,-25,5)$ & $(-50,-30,-25,5)$ & -25 & 5169.097222 & 1 \\
\hline 13 & $(-40,-25,-25,0)$ & $(-40,-25,-25,0)$ & -22.5 & 3338.958333 & 2 \\
\hline 14 & $(-30,-15,-15,0)$ & $(-30,-15,-15,0)$ & -15 & 121.875 & $* * *$ \\
\hline 15 & $(-20,-5,-5,10)$ & $(-20,-5,-5,10)$ & -10 & 121.875 & 6 \\
\hline 16 & $(-30,-15,-5,10)$ & $(-30,-15,-5,10)$ & -5 & 121.875 & 3 \\
\hline
\end{tabular}

*** denote tied values

Since all differences are positive, the value of $\mathrm{T}^{+}$(sum of positive signed rank) is 66 and the standardized test statistic $\mathrm{Z}$ is obtained as 2.934. The corresponding p-value (assuming two sided alternative) is found to be 0.003 . Thus at $1 \%$ level the hypothesis $H: \tilde{\mu}=(70,80,80,90)$ is rejected.

\section{Conclusion}

A new approach to test hypothesis about the median of a population using Wilcoxon signed rank test is discussed in this paper when the underlying observations take imprecise/fuzzy values. The proposed test orders fuzzy numbers based on their expected values and variances using the concepts available in credibility theory. This method of ordering fuzzy numbers is easy to implement when compared to other existing methods that make use of $\alpha$-cut approach for performing arithmetic and relational operations. Steps involved in computation of the proposed test are explained through numerical illustration by assuming the observations to be trapezoidal fuzzy numbers. Further research is being carried out to extend other non-parametric tests for imprecise observations.

\section{Acknowledgment}

The author thank the University Grants Commission, New Delhi, India for providing financial assistance under Minor research project scheme (F.No:41-1396/2012) to carry out the above research work.

\section{References}

[1] Lehmann, E.L. and Romano, J.P., Testing statistical hypothesis (Springer, 2005)

[2] Gibbons, J.D. and Chakraborti, S., Nonparametric statistical inference (Marcel Dekker, New York, 2003, $4^{\text {th }}$ edition).

[3] Zadeh, L.A., Fuzzy sets, Information and Control, 8(3), 1965, 338-353.

[4] Kruse, R., Statistical estimation with linguistic data, Information Sciences, 1984, 197-207.

[5] Kruse, R. and Meyer, K.D., Statistics with vague data (Reidel, Dordrecht, 1987)

[6] Manton, K.G., Woodbury, M.A. and Tolley, D.H., Statistical applications using fuzzy sets (Wiley, New York, 1994).

[7] Viertl, R., Statistical methods for fuzzy data (Wiley, Chichester, 2011).

[8] Casals, M.R., Gil, M.A. and Gil, P., The fuzzy decision problem: An approach to the problem of testing statistical hypotheses with fuzzy information, Euro. J. Oper. Res., 27, 1986b, 371-382.

[9] Romer, C. and Kandel, A., Statistical tests for fuzzy data, Fuzzy Sets and Systems, 72, 1995, 1-26.

[10] Grzegovzewski, P., Testing fuzzy hypothesis with vague data, In C. Bertoluzzi (Ed.), Statistical Modelling, Analysis and Management of fuzzy data, (Physica-Verilag, Heidelberg, 2002) 213-225,

[11] Chen, Y.Y., Statistical inference based on Possibility and belief measures, Trans. Amer. Math .Soc, 347, 1995, 1855-1863

[12] Liu, B., Uncertainty theory (Springer-Verlag, Berlin, 2007).

[13] Vaidyanathan, V.S., Correlation of triangular fuzzy variables using Credibility Theory, International Journal of Computational Cognition, 8(1), 2010, 21-23.

[14] Hesamian, G and Taheri, S.M., Credibility theory oriented Sign test for imprecise observations and imprecise hypotheses, Advances in Intelligent systems and Computing, 190, 2013, 153-163.

[15] Taheri, S.M. and Hesamian, G., A generalization of the Wilcoxon signed-rank test and its applications, Statistical Papers, 54, 2013, 457-470.

[16] Li, X., Tang, W. and Zhao, R., Ranking fuzzy variables by expected value and variance, Proc. $6^{\text {th }}$ International Conference on Fuzzy systems and Knowledge Discovery, 2009, 373 - 377.

[17] Rajagopalan, M. and Dhanavanthan, P., Statistical inference (PHI Learning Pvt. Ltd., New Delhi, 2012 